\title{
Observations of lower hybrid cavities in the inner magnetosphere by the Cluster and Viking satellites
}

\author{
A. Tjulin ${ }^{1,2, *}$, M. André ${ }^{1,2}$, A. I. Eriksson ${ }^{1,2}$, and M. Maksimovic ${ }^{3}$ \\ ${ }^{1}$ Swedish Institute of Space Physics, Uppsala, Sweden \\ ${ }^{2}$ Department of Astronomy and Space Physics, Uppsala University, Sweden \\ ${ }^{3}$ LESIA, Observatoire de Paris, Meudon, France \\ *now at: LPCE/CNRS, 3A, avenue de la Recherche Scientifique 45071 Orléans, France
}

Received: 8 December 2003 - Revised: 28 May 2004 - Accepted: 9 June 2004 - Published: 7 September 2004

\begin{abstract}
Observations by the Viking and Cluster satellites at altitudes up to $35000 \mathrm{~km}$ show that Lower Hybrid Cavities (LHCs) are common in the inner magnetosphere. LHCs are density depletions filled with waves in the lower hybrid frequency range. The LHCs have, until recently, only been found at altitudes up to $2000 \mathrm{~km}$. Statistics of the locations and general shape of the LHCs is performed to obtain an overview of some of their properties. In total, we have observed 166 LHCs on Viking during $27 \mathrm{~h}$ of data, and 535 LHCs on Cluster during $87 \mathrm{~h}$ of data. These LHCs are found at invariant latitudes from the auroral region to the plasmapause. A comparison with lower altitude observations shows that the LHC occurrence frequency does not scale with the flux tube radius, so that the LHCs are moderately rarer at high altitudes. This indicates that the individual LHCs do not reach from the ionosphere to $35000 \mathrm{~km}$ altitude, which gives an upper bound for their length. The width of the LHCs perpendicular to the geomagnetic field at high altitudes is a few times the ion gyroradius, consistent with observations at low altitudes. The estimated depth of the density depletions vary with altitude, being larger at altitudes of $20000-35000 \mathrm{~km}$ (Cluster, 10-20\%), smaller around 1500-13000 km (Viking and previous Freja results, a few percent) and again larger around $1000 \mathrm{~km}$ (previous sounding rocket observations, 1020\%). The LHCs in the inner magnetosphere are situated in regions with background electrostatic hiss in the lower hybrid frequency range, consistent with investigations at low altitudes. Individual LHCs observed at high altitudes are stable at least on time scales of $0.2 \mathrm{~s}$ (about the ion gyro period), which is consistent with previous results at lower altitudes, and observations by the four Cluster satellites show that the occurrence of LHCs in a region in space is a stable phenomenon, at least on time scales of an hour.
\end{abstract}

Key words. Magnetospheric physics (auroral phenomena; plasma waves and unstabilities) - Space plasma physics (general or miscellaneous)

Correspondence to: A. Tjulin

(tjulin@cnrs-orleans.fr

\section{Introduction}

Localized wave packets in the lower hybrid frequency range were first observed by LaBelle et al. (1986) in data from the Marie sounding rocket. Similar features have later been observed on several other sounding rockets (e.g. Kintner et al., 1992; Vago et al., 1992; Pinçon et al., 1997; Lynch et al., 1999; Knudsen et al., 1999) and on the Freja satellite (e.g. Eriksson et al., 1994; Pécseli et al., 1996; Dovner et al., 1997; Høymork et al., 2000), all between $700 \mathrm{~km}$ and $1800 \mathrm{~km}$ altitude. As the wave intensification generally coincides with a density depletion of the same perpendicular size (a few ion gyroradii), we use the term "Lower Hybrid Cavities" (LHC) to denote this phenomenon. Another common name is "Lower Hybrid Solitary Structure" (LHSS). The theory and observations of LHCs have recently been reviewed by Schuck et al. (2003).

A statistical examination of the Freja data (Dovner et al., 1997) showed that the LHCs were mostly distributed in two regions. One region was the auroral oval, including the dayside cusp, with no preference in local time. The other region was at invariant latitudes between $55^{\circ}$ and $65^{\circ}$ in the morningside sector, at magnetic local times between 8 and 12. Furthermore, the LHCs were usually observed in groups from a few tens to several hundreds, and they seemed to be closely associated with background wave activity in the lower hybrid frequency range.

Another statistical survey of Freja data by Høymork et al. (2000) showed that a cylindrical Gaussian is a good model for the density depletion and that the depth of this depletion rarely exceeds $10 \%$ of the background plasma density. There have also been higher altitude satellite observations of LHCs. Pottelette et al. (1992) observed a structure with the Viking satellite that they interpreted as collapsing lower hybrid caviton. This structure does not closely resemble the LHCs identified in the ionosphere, which convincingly have been shown not to be collapsing cavitons (Pécseli et al. (1996)). However, LHCs similar to the ionospheric observations have recently been found in the data sets from the Viking and Cluster satellites (Tjulin et al., 2003). The Viking LHCs were found in the 


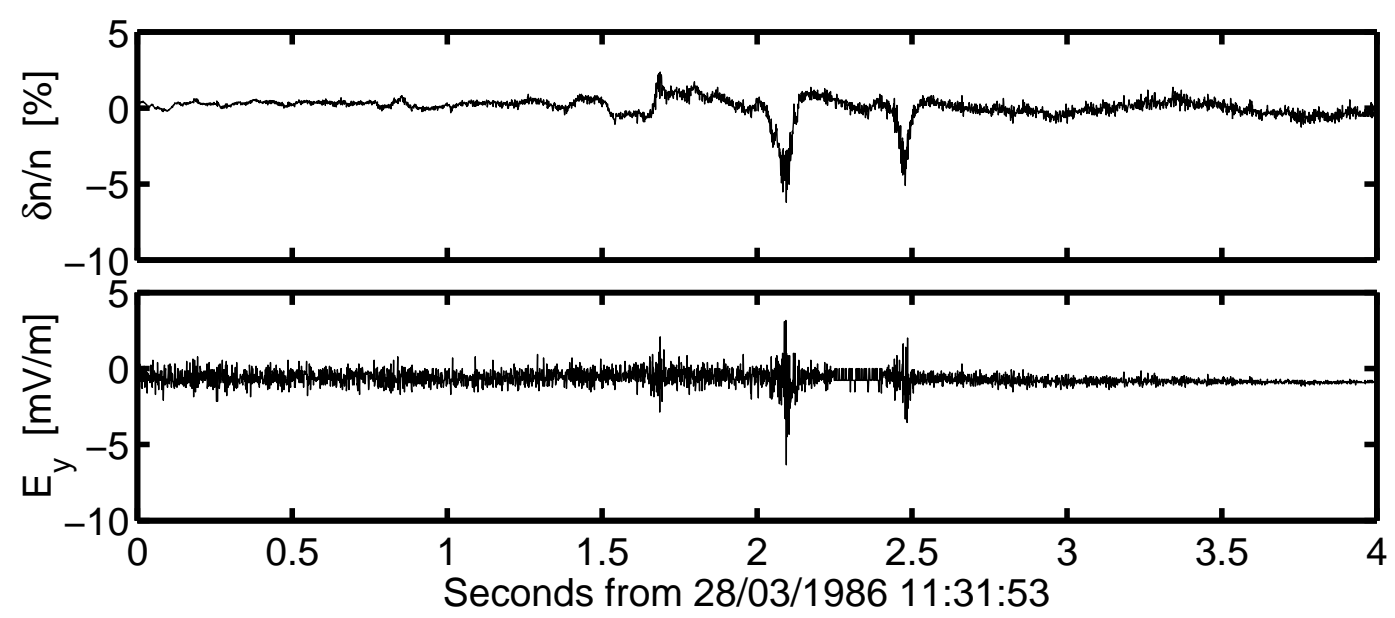

Fig. 1. Typical example of lower hybrid cavities in Viking data. The upper panel shows the density variation and the lower panel the electric field. The electric field amplitude is probably underestimated due to undersampling.

inner magnetosphere at altitudes up to $13000 \mathrm{~km}$, and a Cluster LHC was found at an altitude of $23500 \mathrm{~km}$. The purpose of the present study is to expand the Viking and Cluster observations by Tjulin et al. (2003). By scanning all available data in relevant data taking modes, we establish the occurrence frequency of LHCs at higher altitudes, and study the altitude dependence of the cavity depths and widths. We also compare the background lower hybrid hiss with observations from lower altitudes. By using observations of an LHC region by all four Cluster satellites, we investigate the stability of a region where LHCs are found.

\section{Instrumentation and LHC identification}

The identification of LHCs is performed differently on the Viking and Cluster satellites depending on the instrumentation on board. Below follows a description of the instruments and how the identification is done.

\subsection{Viking}

The Viking satellite (Hultqvist, 1990) was launched in 1986 into a polar orbit with perigee at $817 \mathrm{~km}$ and apogee at $13530 \mathrm{~km}$ altitude. We have used data from the V4L lowfrequency wave instrument (Eriksson et al., 1997). This instrument uses four spherical Langmuir probes mounted on wire booms with $40 \mathrm{~m}$ tip-to-tip distance, and samples two waveforms simultaneously at 428 or 856 samples/s. The waveforms can either be relative density variations $(\delta n / n)$, estimated from the probe current variations from a single probe when the probe is at a fixed voltage with respect to the satellite (Eriksson and Boström, 1998), or the electric field, estimated from the potential difference between two opposite probes with the same bias current. In addition to these measurements, the probe current to the probes in density mode is also sampled, at 107 samples/s. This can be used to estimate the background density of the plasma. We also use some supporting data from the electric field filter banks of the V4L and V4H instruments (Bahnsen et al., 1988), for the study of waves of higher frequencies.

To identify an LHC, we need measurements of both the density variations and the wave electric field at a sufficient sampling rate. For the range of lower hybrid frequencies encountered in the regions scanned by Viking, this means that we have to confine ourselves to data sampled at 856 samples/s, with one channel measuring $\delta n / n$ and the other measuring the electric field. This makes the total data set available rather small: in total we can only use 12 orbits out of Viking's total fully operational time of about one year. All these orbits have been found between March and May 1986. An example of LHCs in the Viking data is shown in Fig 1 . The upper panel shows the density variation and the lower panel the electric field.

Density depletions are readily found in the Viking data by visually inspecting the $\delta n / n$ data. In the upper panel of Fig. 1, we see two clear examples. In order to identify these depletions as LHCs, we check if these density variations are corresponding to increases of at least $25 \%$ in the wave electric field. From the lower panel, it is evident that the amplitude of the wave electric field is larger at the cavities, and hence we have identified two LHCs. Even at 856 samples/s, the time resolution of the electric field wave form measurements is often too low to fully resolve waves near the typical lower hybrid frequency of $1 \mathrm{kHz}$ at Viking altitudes, but by using a combination of undersampled wave form data and filter bank data, the LHCs can still be unambiguously identified, particularly as previous studies have shown trapped waves well below the local lower hybrid frequency inside LHCs (Pécseli et al., 1996; Pinçon et al., 1997; Tjulin et al., 2003). Only density depletions in the Viking data with duration less than $0.5 \mathrm{~s}$ have been considered candidates for being LHCs which corresponds to a cavity width less than about $2 \mathrm{~km}$. The widest confirmed LHC was observed during $0.33 \mathrm{~s}$. In order to detect the increase in wave activity, we 
need at least about 10 samples of the electric field time series, which corresponds to about $0.012 \mathrm{~s}$ when the sampling frequency is $856 \mathrm{samples} / \mathrm{s}$. This gives a lower limit to the observable widths. Density depletions that are smaller than $0.5 \%$ are difficult to detect in the data because of background noise, so this value is used as a lower limit for identifying LHCs.

\subsection{Cluster}

The Cluster mission (Escoubet et al., 1997, 2001) consists of four identical spacecraft flying in formation in a polar orbit with perigee at $19000 \mathrm{~km}$ and apogee at $119000 \mathrm{~km}$. Thanks to this wide variation in altitude, Cluster will be in several different environments during the orbit, and so it is possible to look for LHCs in regions with a large range of plasma parameters. In order to limit the study, we only consider data from the inner magnetosphere, which we here define to be altitudes up to $40000 \mathrm{~km}$.

Most of the Cluster data used here are from the Electric Field and Wave (EFW) instrument (Gustafsson et al., 1997). This instrument measures electric fields using two pairs of spherical probes mounted on wire booms in the spin plane of the satellite. Each pair has a probe-to-probe distance of $88 \mathrm{~m}$. The instrument mostly samples the electric field at 25 samples/s, which is insufficient for LHC identification. In this study, we therefore concentrate on data taken in "burst mode" (BM1), when the EFW sampling rate is 450 samples/s with an anti-aliasing filter at $180 \mathrm{~Hz}$. Such data are available for intervals of a few hours, normally occurring once or twice per week. Sometimes shorter periods with much higher time resolution are available and can be used for detailed studies of LHCs (Tjulin et al., 2003).

In addition to the electric field data, the potential of each probe with respect to the spacecraft is routinely sampled at 5 samples/s. From these measurements the spacecraft potential can be estimated, and thus also the plasma density (Pedersen, 1995; Pedersen et al., 2001). However, individual LHCs are often observed during less than $0.2 \mathrm{~s}$ in Cluster data, and hence routine EFW estimates of the density do not have the time resolution to detect these cavities. In order to correctly identify LHCs, we need to have at least one measurement of a single-probe potential at 450 samples/s. Due to telemetry limitations, we usually only have differential probe voltages (electric field) measurements at this high sampling frequency when in burst mode, but following failures of one of the probes on each of spacecraft 1 (28 December 2001) and spacecraft 3 (29 July 2002), such data have been available from these spacecraft in almost all burst mode intervals.

In order to study waves at higher frequencies in and around LHCs, data from the Spectrum Analyzer of the SpatioTemporal Analysis of Field Fluctuations (STAFF-SA) instrument (Cornilleau-Wehrlin et al., 1997) are also used. This instrument provides on-board analysis of three magnetic and two electric wave field components up to $4 \mathrm{kHz}$. The background magnetic field is determined using data from the
FluxGate Magnetometer (FGM) instrument (Balogh et al., 1997).

An example of typical Cluster LHCs is shown in Fig. 2. Here the upper panel shows the potential of probe 2 with respect to the spacecraft measured at $450 \mathrm{samples} / \mathrm{s}$. A decrease in this potential indicates a decrease in the density. The middle panel displays the electric field measured between probes 3 and 4, also sampled at $450 \mathrm{~Hz}$. Here the spacecraft spin period of 4 seconds is clearly seen. While the upper two panels show data from the EFW instrument, the bottom panel displays a STAFF-SA electric field spectrogram for frequencies up to $4 \mathrm{kHz}$. The upper panel indicates several density cavities lasting about $0.2 \mathrm{~s}$. The background density is here about $33 \mathrm{~cm}^{-3}$ (corresponding to around $3.5 \mathrm{~V}$ ) while the deepest cavity after $7 \mathrm{~s}$ has a relative decrease of $25 \%\left(25 \mathrm{~cm}^{-3}\right.$ at $\left.-4 \mathrm{~V}\right)$. To identify a density cavity as an LHC, we also require an increase of the electric field amplitude in the lower hybrid frequency range. The 12 confirmed LHCs in Fig. 2 are indicated with dotted lines.

In our statistical search for LHCs in the Cluster data, we have required that a density depletion of at least about $2 \%$ should coincide with a clear increase of the electric field wave amplitude in the lower hybrid frequency range, at least $25 \%$. The limit of the density depletion comes from difficulties to distinguish cavities smaller than $2 \%$ from background noise. This is not as high a level of sensitivity as we have used for Viking, where a different technique (Langmuir probe at voltage bias) allowed going down to $0.5 \%$ depletions (see Sect. 2.1 above).

In the regions from which we use Cluster data in this study, the lower hybrid frequency is typically around $250 \mathrm{~Hz}$. Since the EFW electric field data is sampled at 450 samples/s, waves above the lower hybrid frequency can often not be observed with this instrument.

There are still two ways to identify waves within LHCs. First, as noted above, we should find trapped modes well below the lower hybrid frequency inside the cavities. This increase of lower frequency waves is often easy to identify in EFW data, since most of the background hiss emissions at higher frequencies are removed by the anti-aliasing filter. The middle panel of Fig. 2 shows clear increases in the electric field wave data, coinciding with many of the density cavities. Second, in the burst mode intervals, the STAFF-SA electric field spectra often has the time resolution of $0.25 \mathrm{~s}$, which is sufficient to identify waves associated with the density cavities, also well above the lower hybrid frequency. This is clearly seen in the bottom panel of Fig. 2 .

Often the EFW wave form data for lower frequencies are sufficient to decide that a density cavity is an LHC, and STAFF-SA spectrograms covering higher frequencies can be used for confirmation, but for some cavities the STAFF-SA high frequency data are needed for the identification (see the two cavities about $2.5 \mathrm{~s}$ into the interval in Fig. 2). Only cavities measured during less than $2 \mathrm{~s}$ were considered to be potential LHCs, and the widest confirmed LHC was observed during $1.4 \mathrm{~s}$. The time resolution of the instrument gives a 


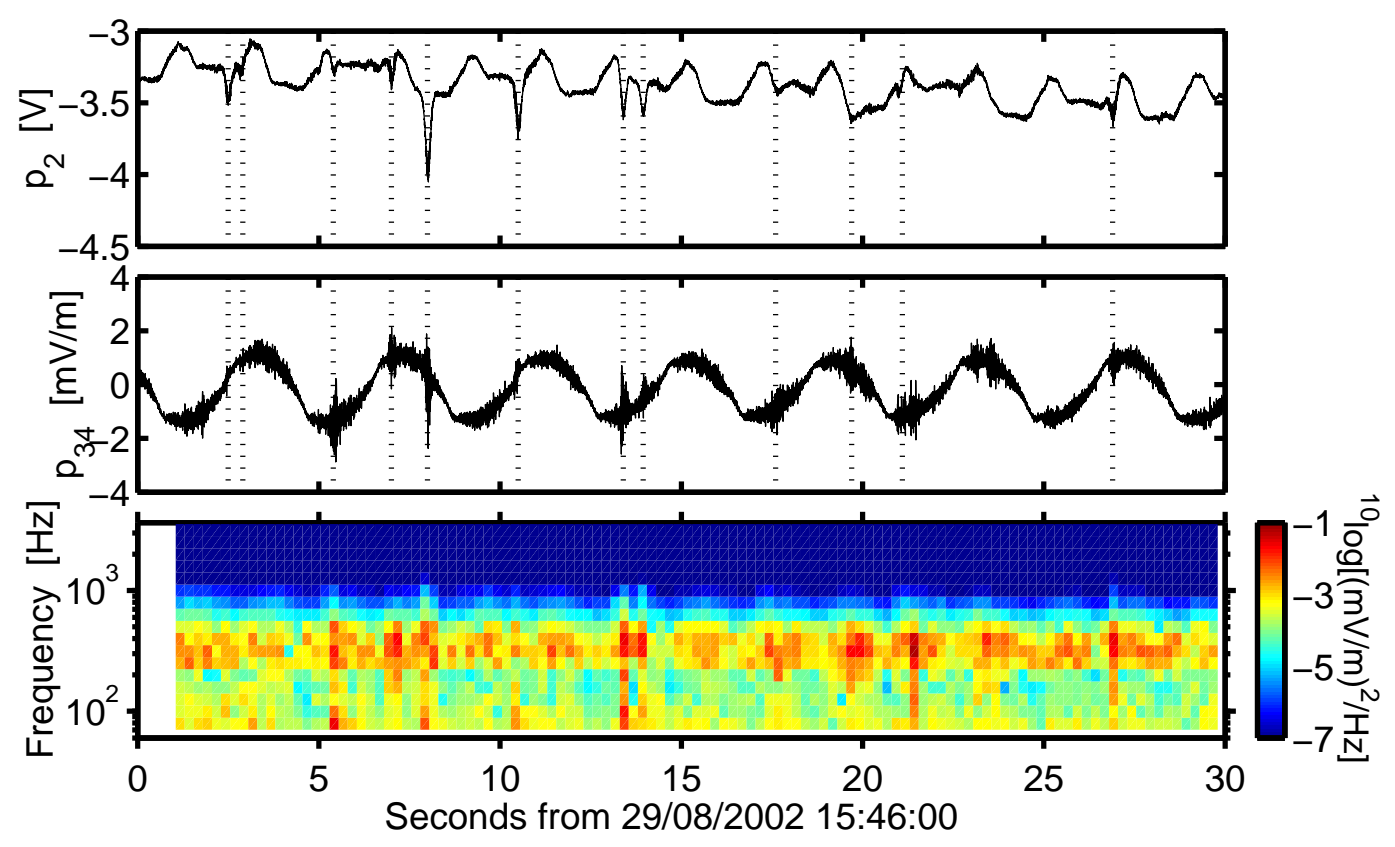

Fig. 2. Typical example of LHCs observed by Cluster 1. The upper panel shows high resolution observations of the potential of probe 2 with respect to the spacecraft, which can be used as an estimate of the density (a decrease in this parameter indicates a decrease in the density). The regular increases of this potential every $2 \mathrm{~s}$ are caused by asymmetries in the spacecraft and the $4 \mathrm{~s}$ spin. The middle panel displays the electric field measured between probes 3 and 4 with a $180 \mathrm{~Hz}$ low-pass filter. The satellite spin is obvious in this panel. The bottom panel gives an electric field spectrogram up to $4 \mathrm{kHz}$. The lower hybrid frequency is about $240 \mathrm{~Hz}$, and the identified LHCs are indicated by dotted lines.

lower limit to the observable widths of about $0.02 \mathrm{~s}$, which typically corresponds to about $100 \mathrm{~m}$ along the satellite path.

\section{Location of the LHCs}

It is of interest to see where the LHCs are found in the Viking and Cluster data, so that we can compare with occurrence investigations from the Freja satellite (Dovner et al., 1997; Kjus et al., 1998). We also compare how common the LHCs are in the inner magnetosphere with those observations from the upper ionosphere.

\subsection{Viking}

As discussed above, the data set used for the survey of the Viking cavities consists of all data from the V4L instrument during times when one channel is sampling $\delta n / n$ and the other channel is sampling the electric field, both with the best time resolution of 856 samples/s. There were 12 orbits with these favourable settings of the instrument, providing in total $27 \mathrm{~h}$ of data. Lower hybrid cavities were found on 7 of these orbits, and a total number of 166 individual LHCs were identified.

The examined Viking orbits are shown in Fig. 3, which is a diagram with Magnetic Local Time (MLT) and invariant latitude. The locations of the individual identified LHCs are marked with dots in this diagram. All examined orbits are from the Northern Hemisphere. The examined orbits cover only a small part of the diagram, so it is difficult to say something conclusive about the occurrence of LHCs but the amount of data is at least sufficient to obtain an overview of the locations.

The distribution of the identified Viking LHCs with invariant latitude is shown in Fig. 4, where the occurrence frequency is the number of LHCs found per five minutes of observation time in the given latitude range. Five minutes typically corresponds to about $1000 \mathrm{~km}$ along the satellite path. Most of the Viking cavities were found at invariant latitudes below $70^{\circ}$, and no cavity was found below $60^{\circ}$. The occurrence frequency goes down with increasing invariant latitude.

\subsection{Cluster}

The search for LHCs in the Cluster data was performed using all EFW data where the spacecraft potential was measured with 450 samples/s resolution, i.e. from 28 December 2001 on spacecraft 1 and 29 July 2002 on spacecraft 3, as described in Sect. 2.2. In this study, we use data up to 24 April 2003, when the study was initiated. In addition, since the scope here is to study phenomena that had previously only been observed at lower altitudes, only data taken close to perigee of the rather eccentric Cluster orbits are taken into account. The total data set therefore consists of 45 Cluster burst mode data intervals, or $87 \mathrm{~h}$ of data in total. LHCs were found on 32 of these 45 passages, and a total number of 535 individual LHCs were identified. 


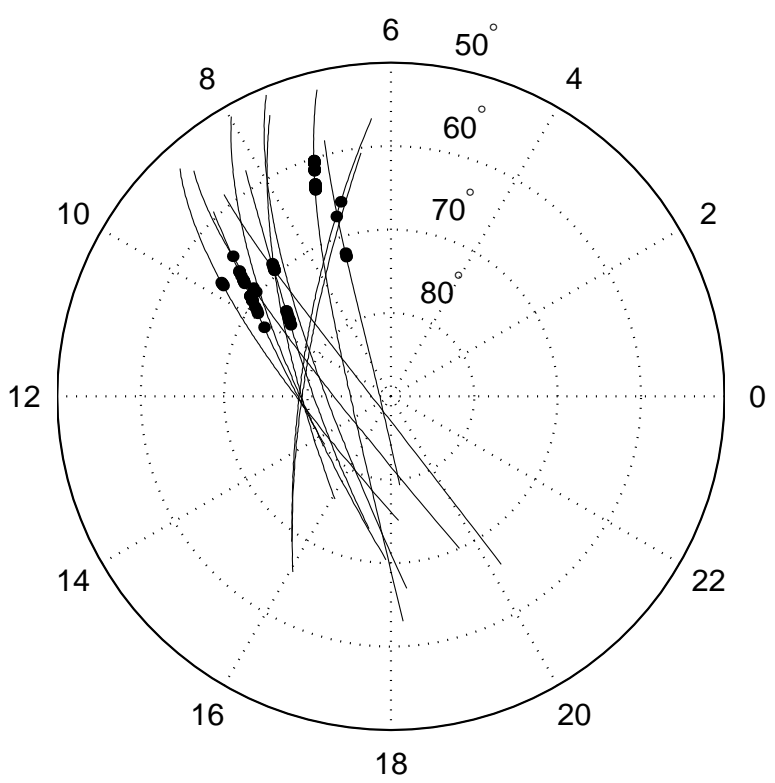

Fig. 3. Diagram with Magnetic Local Time (MLT) and invariant latitude, showing the investigated data intervals (the lines) and the location of the identified LHCs (the dots) in the Viking data set. The total number of LHCs is 166, so many of the dots in the figure overlap. The altitudes of the orbits are shown in Fig. 7.

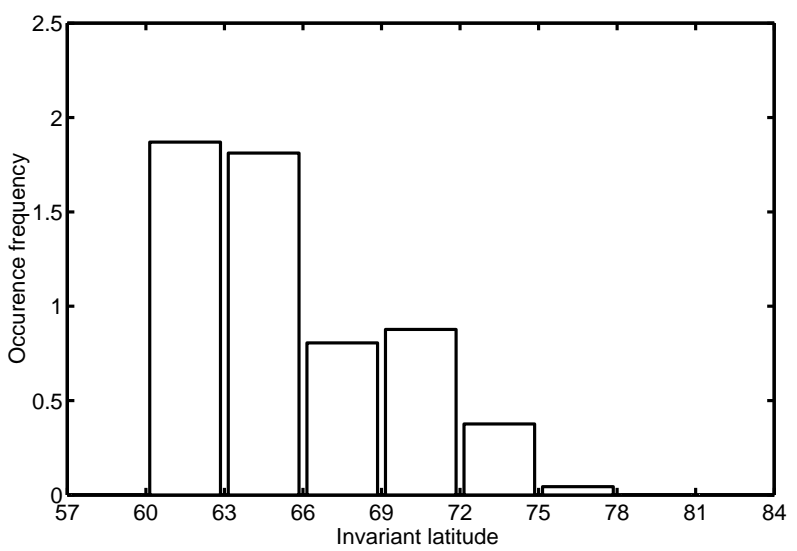

Fig. 4. Distribution of the invariant latitude of the identified Viking LHCs. The normalisation used here is the number of LHCs found per five minutes in the latitude range of interest. Five minutes typically corresponds to about $1000 \mathrm{~km}$ along the satellite path.

The examined orbits of the Cluster spacecraft are displayed in Fig. 5. This is the same kind of diagram as for the Viking orbits (Fig. 3), covering MLT and invariant latitude. The examined Cluster orbits are more evenly spread in the diagram than the Viking orbits, but the data are still not very evenly distributed in MLT, with almost no data taken between 04:00 and 08:00 MLT and much data at magnetic noon. The polar orbits of the Cluster spacecraft also introduce a seasonal dependence of the MLT coverage, since all data are from the Northern Hemisphere. The orbits are such that 18:00 MLT is covered around midsummer and 12:00 MLT

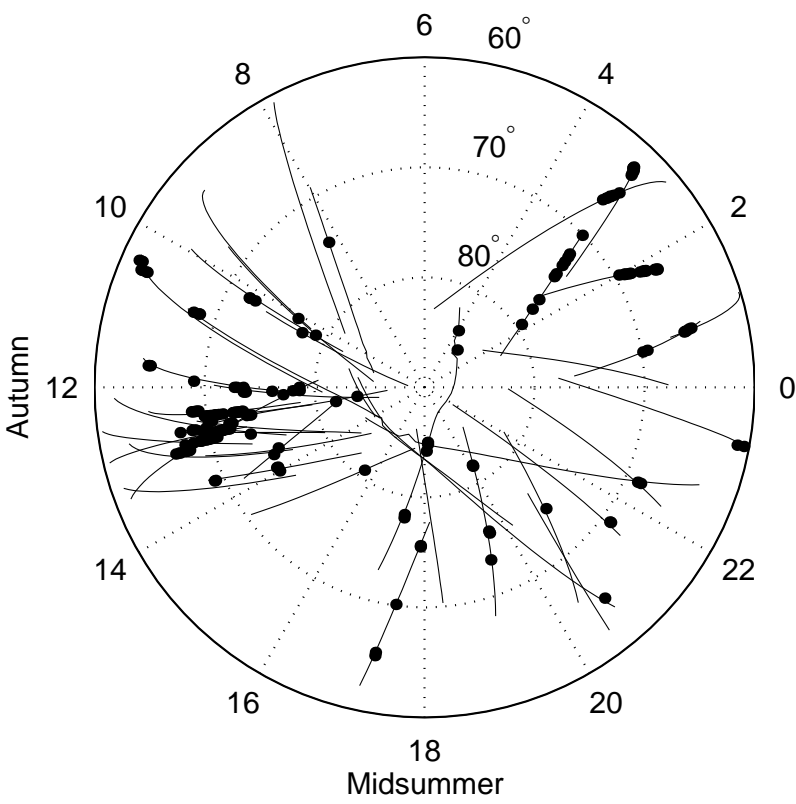

Fig. 5. Diagram with Magnetic Local Time (MLT) and invariant latitude, showing the investigated data intervals (the lines) and the location of the identified LHCs (the dots) in the Cluster data set. The total number of identified LHCs is 535, so many of the dots in the figure overlap. The altitudes of the orbits are shown in Fig. 7.

during autumn. This may be of some importance if LHCs depend on ionospheric conditions, which vary with season. Despite these sources of bias, the statistics provide an overview of where we may find LHCs. The locations of the identified LHCs are marked with dots in Fig. 5.

The distributions of the identified Cluster LHCs in invariant latitude and MLT are shown in Fig. 6. The normalisation used here is the same as in the Viking statistics (Fig. 4), the number of LHCs per five minutes in the region of interest, and it typically corresponds to $1400 \mathrm{~km}$ along the spacecraft path when effects of the plasma drift are not included. We see that the highest occurrence frequency of the Cluster cavities is found between $60^{\circ}$ and $76^{\circ}$ invariant latitude, but we must note that the orbits of the Cluster spacecraft do not reach invariant latitudes below $60^{\circ}$. Furthermore, the highest occurrence frequencies are found at noon or midnight magnetic local time, but because of the uneven distribution of orbits in MLT, the occurrence frequencies for MLT between 4 and 8 are very uncertain and are therefore not included in the figure.

\subsection{Discussion of occurrence statistics}

The investigated data intervals may also be displayed in terms of the altitude as a function of the invariant latitude or the MLT. This is done in Fig. 7, where the investigated Viking orbits are marked with dashed lines and the Cluster orbits with solid lines. The location of the identified LHCs are marked with dots. The altitudes of the spacecraft are evidently dependent on the invariant latitude, but not so clearly 

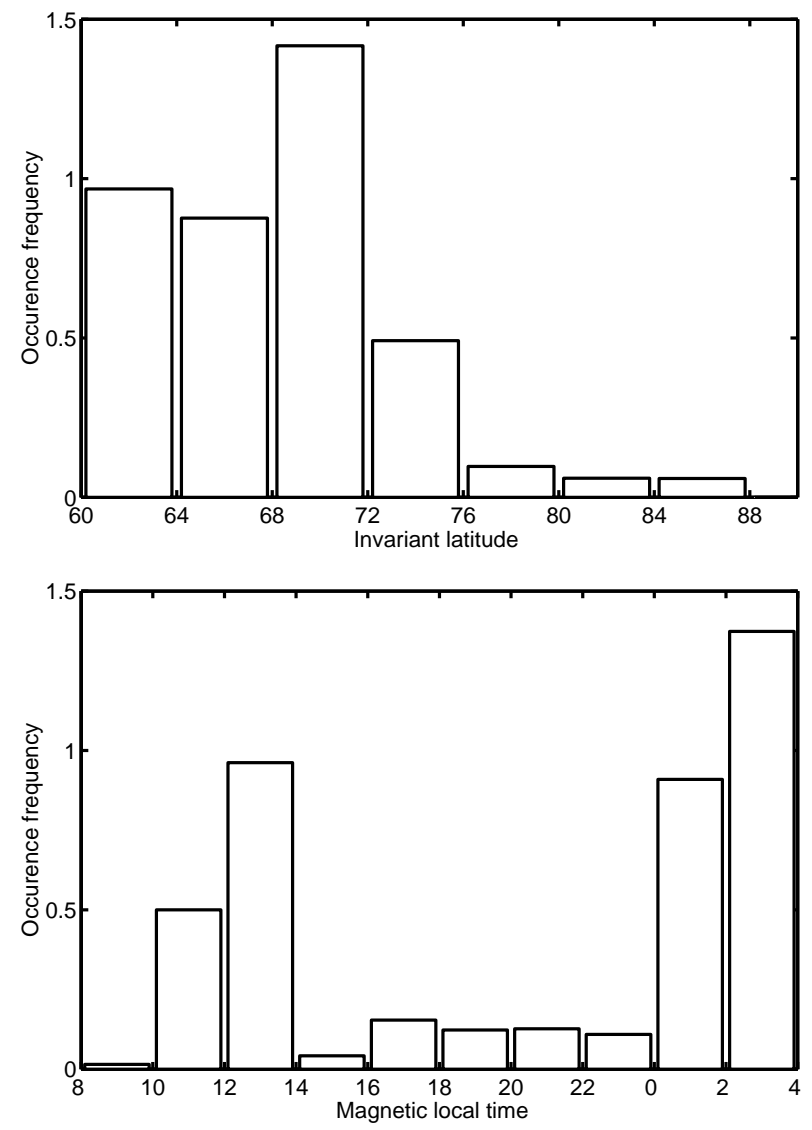

Fig. 6. The distribution of the identified Cluster lower hybrid cavities in invariant latitude (upper panel) and MLT (lower panel). The normalisation is the number of cavities per $5 \mathrm{~min}$ of observation time in the region of interest. Five minutes typically corresponds to about $1400 \mathrm{~km}$ along the spacecraft path. Note that between 04:00 and 08:00 MLT, almost no data were taken, so this MLT interval is not included in the lower panel.

on MLT because of the polar orbits. We may once again point out that the coverage of different MLT is very uneven, especially for the Viking satellite.

In general, the LHCs in this study are found in groups with up to twenty cavities, so the value of the occurrence frequencies in Figs. 4 and 6 give only statistical information. You may, as in the example in Fig. 2, find 12 cavities within $30 \mathrm{~s}$, or no cavities in one hour. The LHCs found here are at invariant latitudes from the auroral region, including the dayside cusp, down to the plasmapause. A common feature of the LHC distributions for the Viking (Fig. 4) and Cluster (Fig. 6) data sets is that the occurrence frequency goes down with increasing invariant latitude. The absence of LHCs below invariant latitude $60^{\circ}$ in the Viking observations cannot be verified by the Cluster observations because the Cluster orbits never reach to such low invariant latitudes. There is a peak at about $70^{\circ}$ invariant latitude in the Cluster data, and it can also be seen in the Viking data, but not as clearly, as a disruption in the trend of decreasing occurrence frequency for larger invariant latitudes. Although our data set is not as
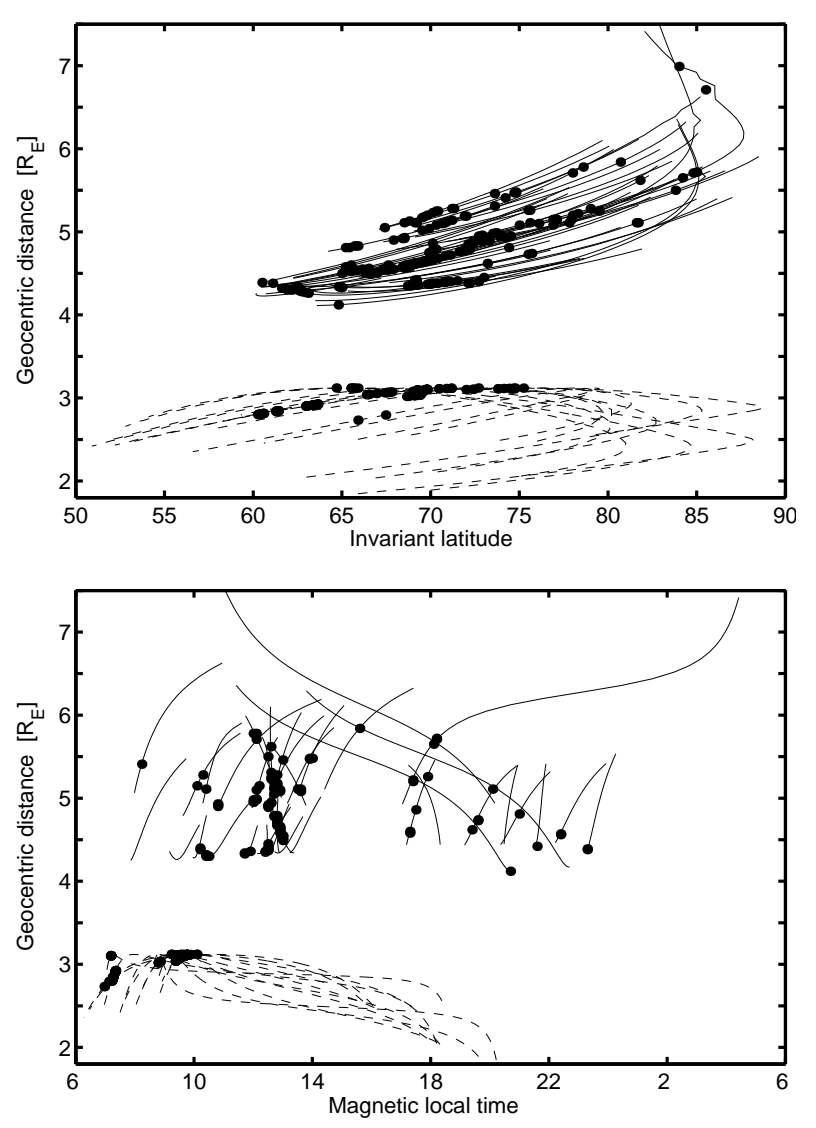

Fig. 7. The geocentric distance as a function of invariant latitude (upper panel) and MLT (lower panel) for the investigated data intervals for Cluster (the solid lines) and for Viking (the dashed lines). The dots mark the locations of the identified LHCs. There are in total 701 identified LHCs, so many of the dots overlap.

large as the Freja data set used for previous statistical investigations at low altitudes, we may do some comparisons.

First, statistical analysis of data from the Freja satellite has shown that the LHCs in the upper ionosphere are mostly either in the auroral oval without preference in MLT, or at invariant latitudes between $55^{\circ}$ and $65^{\circ}$ and at MLT between 8 and 12 (Dovner et al., 1997). Our observations at higher altitude do not conflict with these results, though it appears that we can see hints of a concentration just after rather than just before magnetic noon.

Since the LHCs are supposed to be structures elongated along the magnetic field (Vago et al., 1992), it is useful to determine the LHC occurrence frequency in terms of the number of LHCs per kilometer of the satellite path perpendicular to the magnetic field. For the Cluster observations we have about 0.001 LHCs per kilometer for the invariant latitudes with most LHC activity. An estimate for the Viking observations gives about 0.02 identified LHCs per kilometer along the satellite orbit. These are values in an inertial frame of reference. On Cluster, the plasma drift speed generally dominates over the spacecraft speed in this frame, so that the number of LHCs encountered in the plasma reference frame 
Table 1. Comparison of some parameters relevant for the occurrence frequency of the LHCs. The occurrence density is the number of LHCs per unit length perpendicular to the magnetic field, and the occurrence frequency is the number of LHCs per normalised flux tube radius. All parameters are normalised to the Freja numbers.

\begin{tabular}{cccc}
\hline Spacecraft & Occ. density & Flux tube radius & Occ. frequency \\
\hline Freja & 1 & 1 & 1 \\
Viking & $1 / 100$ & 4 & 0.04 \\
Cluster & $1 / 2000$ & 10 & 0.005 \\
\hline
\end{tabular}

will be even smaller. We will show in Sect. 4 that the difference in occurrence frequency cannot be due to the slightly different identification criteria used on Viking (Sect. 2.1) and Cluster (Sect. 2.2).

The occurrence frequency for some of the Freja orbits has been determined by Kjus et al. (1998) to be about 2 per kilometer perpendicular to the magnetic field. The LHCs thus were a factor 2000 more common per kilometer in the Freja data compared to Cluster. If most LHCs should reach from a few thousand to many thousand kilometers in length, we would expect that the occurrence frequency per flux tube width should be constant with altitude. The radius of a flux tube, however, is only about 10 times larger at Cluster altitudes compared with the Freja. The Viking LHC occurrence frequency is a factor of 100 lower, and the flux tube radius a factor of 4 larger, compared to Freja. These observations are put together in Table 1, where we see that the relative occurrence frequency decreases with altitude. It is not clear what causes this decrease. The necessary background VLF waves could be more common at lower latitude, or these waves could for some reason accumulate more easily to form LHCs in the denser low-altitude plasmas. As LHCs are less abundant at Viking and Cluster altitudes than expected from simple field line scaling from Freja height, one may at least infer that individual LHCs do not extend all along a geomagnetic field line from the ionosphere up to $35000 \mathrm{~km}$. This result is by no means surprising, but is in fact the first upper limit to LHC parallel extension that has been presented in the literature.

\section{Widths and depths of the LHCs}

After finding the locations of the LHCs in the Viking and Cluster data sets, we turn our attention to the individual cavities. Their depths and widths are determined, and the statistics are compared to results from lower altitudes. The depth is the difference between the density at the centre of the LHC and the density just outside it, and the width is here the full width at $10 \%$ of the maximum density depth.
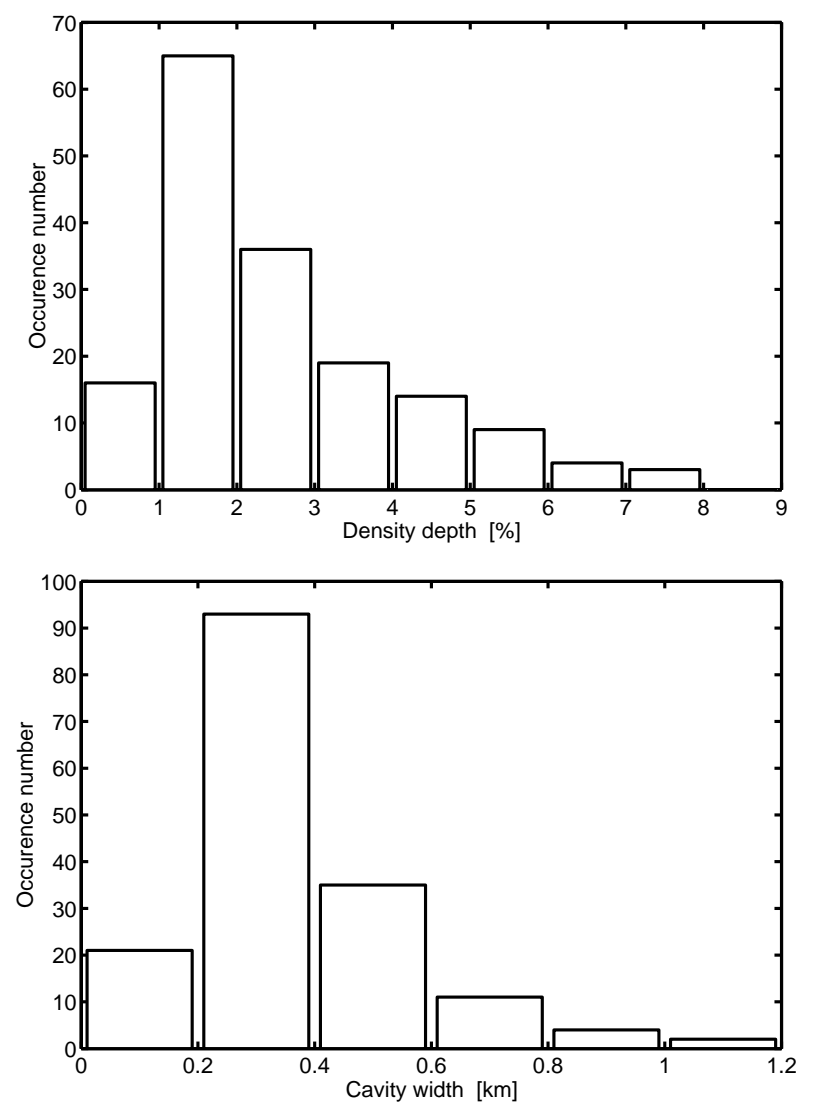

Fig. 8. The distribution of the depths and widths of the identified Viking LHCs. The widths are determined under the assumption that the spacecraft passes the cavity with the typical perpendicular velocity of $3.5 \mathrm{~km} / \mathrm{s}$.

\subsection{Viking}

The depths of the Viking LHCs are given directly by the $\delta n / n$ measurements of the V4L instrument, while the widths are determined by the time it takes for the Viking satellite to traverse them. The speed of the Viking satellite is typically between 3 and $4 \mathrm{~km} / \mathrm{s}$ in this region, which is generally higher than the $\boldsymbol{E} \times \boldsymbol{B}$ drift speed in this region. Under the assumption that the spacecraft velocity is perpendicular to the magnetic field during the LHC crossings, we can estimate an approximate width of the LHCs. The distributions of depths and widths are shown in Fig. 8. The depths can only be considered as a lower limit of the actual LHC depth, since the satellite generally does not pass through the centre of the cavity (Pécseli et al., 1996). They may still serve as a guideline, though.

From Fig. 8, we see that all identified Viking LHCs have depths smaller than $8 \%$. The occurrence number of LHCs with really small depth is very low, but this may be an effect of the difficulty in identifying those LHCs. On the other hand, it has been shown by Tjulin et al. (2004) that the depth of an LHC cannot be arbitrarily small in order for LHCs to exist, so this should not be a problem. It may also be more difficult to observe the smallest LHC widths, since the 

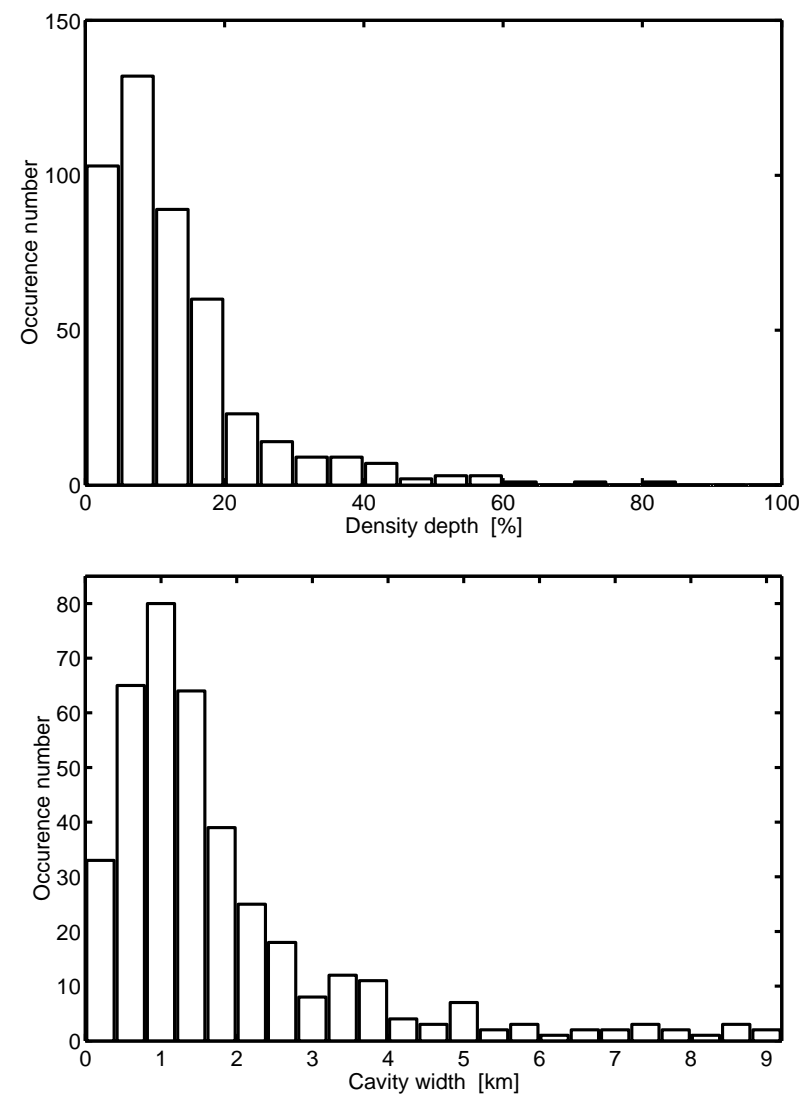

Fig. 9. The distribution of the depths and widths of the identified Cluster LHCs. The widths are determined using the plasma velocity relative to the spacecraft, calculated from the measured electric and magnetic fields.

satellite may pass the cavity too fast to see the density depletion in the data, but this only applies to widths smaller than $40 \mathrm{~m}$. These are not likely to exist, since the typical widths observed by the Freja satellite are $50 \mathrm{~m}$ (Høymork et al., 2000), and the LHC widths are probably larger at higher altitudes since all typical scale lengths increase with altitude.

\subsection{Cluster}

The depths and widths of the identified Cluster LHCs have also been determined. The depths are determined by estimating the plasma density at the minimum of the density depletion as seen by the spacecraft, and the density outside the cavity, using the probe potential (Pedersen, 1995; Pedersen et al., 2001). The values of the measured depths are, of course, dependent both on the actual density depth of the LHC and how close to the centre of the cavity the satellite passes. The upper panel of Fig. 9 shows the distribution of cavity depths for the identified Cluster LHCs. We see that most of the cavities are below $20 \%$ depletion, but some of them are very deep.

The width of an individual LHC is, as for the Viking observations, determined from the time it takes for the satellite to traverse it. The LHCs are elongated along the magnetic field
Table 2. The typical LHC widths determined by different spacecraft. Some typical parameters relevant to the widths are included.

\begin{tabular}{cccc}
\hline Spacecraft & Cavity width & Ion gyroradius & El. inertial length \\
\hline Amicist & $40 \mathrm{~m}$ & $7 \mathrm{~m}$ & $100 \mathrm{~m}$ \\
Freja & $50 \mathrm{~m}$ & $8 \mathrm{~m}$ & $250 \mathrm{~m}$ \\
Viking & $300 \mathrm{~m}$ & $50 \mathrm{~m}$ & $600 \mathrm{~m}$ \\
Cluster & $1000 \mathrm{~m}$ & $600 \mathrm{~m}$ & $1200 \mathrm{~m}$ \\
\hline
\end{tabular}

(Vago et al., 1992), so in order to calculate the width of an LHC, we need to know the plasma velocity perpendicular to the magnetic field, with respect to the satellite. For Viking, we could assume this velocity to be the spacecraft velocity in a geostationary frame, but at Cluster altitudes, the plasma drift speed will usually dominate. Under the assumption that the plasma is infinitely conducting, the plasma drift velocity can be estimated from

$\boldsymbol{v}_{\perp}=\frac{\boldsymbol{E} \times \boldsymbol{B}}{|\boldsymbol{B}|^{2}}$,

where $\boldsymbol{E}$ is the electric field in the spacecraft frame of reference. Three components of the background magnetic field are available from the FGM instrument (Balogh et al., 1997). The two components of the background electric field in the satellite spin plane are estimated, making a satellite spin fit of the electric field measured by the single probe pair measuring the electric field (see Sect. 2.2). The third component of the electric field can be found using $\boldsymbol{E} \cdot \boldsymbol{B}=0$, as long as $\boldsymbol{B}$ is not close to the spin plane. The lower panel of Fig. 9 shows the distribution of widths of the identified Cluster LHCs. In order to keep the errors down on the estimation of the perpendicular velocity, LHCs where the angle between the background magnetic field and the satellite spin plane is less than $5^{\circ}$ are excluded in Fig. 9.

\subsection{Discussion of widths and depths}

The LHCs in the Freja data set had a typical $1 / e$ width of $30 \mathrm{~m}$ (Høymork et al., 2000), which is equivalent to a cavity width of about $50 \mathrm{~m}$, and the LHC width from the AMICIST rocket was about $40 \mathrm{~m}$ (Pinçon et al., 1997). This should be compared with the typical LHC widths from Viking $(300 \mathrm{~m})$ and Cluster (1000 m). In Table. 2, these typical LHC widths are compared with typical values of the ion gyroradius and the electromagnetic skin depth or electron inertial length $c / \omega_{\mathrm{pe}}$, of the plasma in the different regions. We assume that the relevant ions to be hydrogen for Cluster and oxygen for the other spacecraft, and that the ion temperatures are $3 \mathrm{eV}$ for Cluster and Viking, $0.2 \mathrm{eV}$ for Freja (Høymork et al., 2000) and $0.3 \mathrm{eV}$ for AMICIST (Pinçon et al., 1997). The widths of the cavities lie between the ion gyroradius and the electron inertial length for all four spacecraft. One should note that rocket investigations, where the density may vary over several orders of magnitude but the magnetic field 


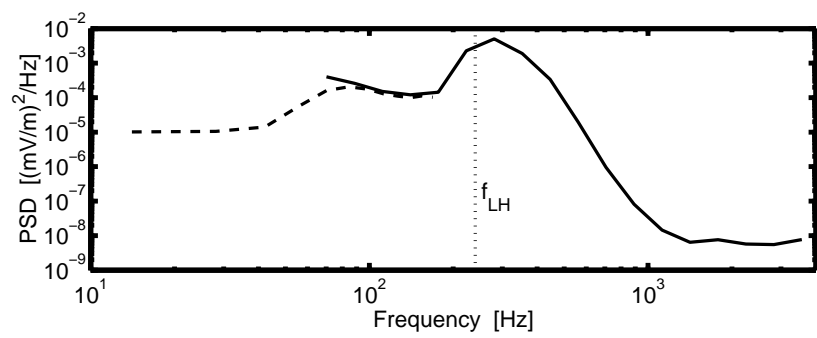

Fig. 10. Spectrum of the background wave electric power spectral density for the Cluster events in the example in Fig. 2. The solid line is obtained using STAFF data and the dashed line is from EFW data. The lower hybrid frequency is indicated for comparison.

strength is essentially constant, have shown no sign of scaling with the skin depth (Knudsen et al., 2003). The apparent consistency with skin depth scaling seen in our study may thus just be a coincidence due to the variation of density with altitude. Scaling with skin depth could be expected if LHCs originate from processes involving inertial Alfvén waves (Shapiro, 1998), while the gyroradius is a natural scale for processes relying on perpendicular ion expulsion (Knudsen et al., 2003). We may also note that the widths also seem to scale with the relative magnetic flux tube radius, given in Table 1.

The estimated depths of the LHCs from the Cluster satellites are in general larger than those from Viking. This could, in principle, be due to the fact that they are determined in different ways. The V4L instrument on Viking used the current measured by Langmuir probes as a density measure, while Cluster EFW uses the spacecraft potential. While this is a difference, each technique should in fact be the more appropriate for the plasma densities encountered around LHCs by that mission. The spacecraft potential is a good indicator of plasma density variations in sufficiently tenuous plasmas (Pedersen, 1995), which in the Cluster case means an electron density below a few hundred per $\mathrm{cm}^{3}$ (Pedersen et al., 2001), but in denser plasmas the spacecraft potential is less sensitive to density variations. On the other hand, variations in current to a Langmuir probe are contaminated by other effects than plasma density fluctuations if the density is too low, so that method is better suited for the denser plasmas encountered by Viking (Eriksson et al., 1997).

Another difference is that Viking V4L only had 8-bit analog-to-digital converters and therefore had to use automatic gain control to cover a large dynamic range, while this is not needed for the 16-bit data from Cluster EFW. However, indications of "clipping" of the wave forms is rare in the Viking V4L data we have used, and we therefore interpret the difference in cavity depth seen on Viking and Cluster as a real variation with altitude.

A comparison with previous observations shows that the typical depth of an LHC in the Freja data was less than $10 \%$ (Høymork et al., 2000). Although density depletions up to $90 \%$ were found on the TOPAZ III rocket (Vago et al., 1992), a major fraction of the observed density depletions

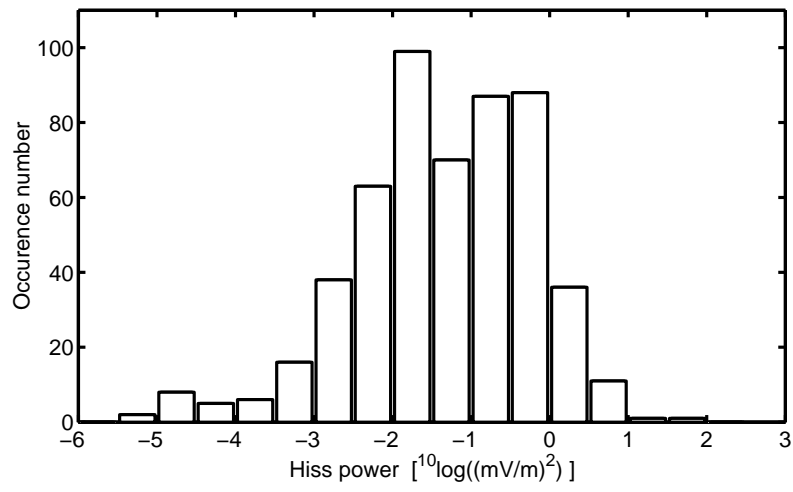

Fig. 11. The distribution of the estimated background lower hybrid hiss wave power for the Cluster LHC events.

were between 10 and 50\% (Kintner et al., 1992). The typical estimated depths of the LHCs thus seem to vary with altitude, with larger depths around $1000 \mathrm{~km}$ (sounding rockets), smaller around 1500-13000 km (Freja and Viking) and again larger at altitudes of $20000-35000 \mathrm{~km}$.

Finally, we may note from the distributions of observed density depletions in Fig. 8 and Fig. 9 that the different minimum values for density depletions accepted as LHCs that we used for Viking $(0.5 \%)$ and Cluster $(2 \%)$ should not significantly influence the occurrence statistics, as none of the distributions peak at the lowest bin in the histograms but show clear signs of a decrease at the lowest bin. The same also holds for the width. We may note that if there are cavities smaller than the ones detected here, they are also smaller than the ion gyroradius.

\section{The background hiss}

The identified LHCs are situated in regions with background lower hybrid hiss; see, for example, the bottom panel of the Cluster example in Fig. 2. The spectrum of the background electric wave emissions in that example is shown in Fig. 10. This spectrum is obtained using both the STAFF and the EFW instruments on data taken during $16 \mathrm{~s}$ immediately after the data in Fig. 2. Since there were no LHCs in this time interval, the spectrum can be considered to show the background. We see a clear enhancement of wave power at frequencies around the lower hybrid frequency, which is typical for lower hybrid hiss. The spectrum in Fig. 10 is similar to observations at lower altitudes.

The total power of the background hiss has been estimated for the Cluster LHCs. This power is here defined as the total integrated wave power at frequencies from the lower hybrid frequency up to four times the lower hybrid frequency, and we have used STAFF electric field spectrum analyzer data for this estimate. The resulting distribution of background hiss wave power is shown in Fig. 11. Some of the LHCs are situated in very weak background hiss but most of the LHCs are found in regions with hiss wave power between 

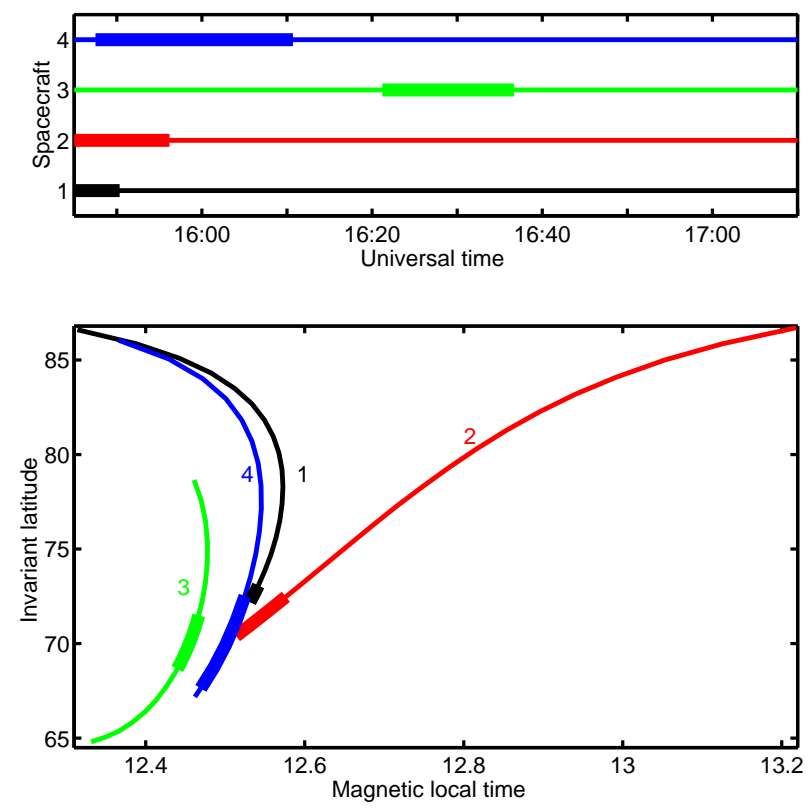

Fig. 12. The four Cluster satellites observing the same region with LHCs at different times. The narrow lines means that we have high resolution (BM1) data, and the thick lines that there are identified LHCs. Note that spacecraft 1 and 2 have LHCs already at the beginning of the time interval.

$10^{-3}(\mathrm{mV} / \mathrm{m})^{2}$ and $10(\mathrm{mV} / \mathrm{m})^{2}$, which is still a very wide range of wave electric power.

Some comparison with rocket data can be of interest here. The wave electric power spectral density for rocket data is typically in an interval between $10^{-4}(\mathrm{mV} / \mathrm{m})^{2} / \mathrm{Hz}$ and $5 \times 10^{-3}(\mathrm{mV} / \mathrm{m})^{2} / \mathrm{Hz}$ (Lynch et al., 1999). To be able to compare this number with the Cluster result, we note that the lower hybrid frequency is typically about $4 \mathrm{kHz}$ in the rocket measurements. If we assume that most of the wave electric power is at frequencies from the lower hybrid frequency to twice that number, see, for example, Fig. 10, we obtain estimates of the total wave electric power that are between $0.4(\mathrm{mV} / \mathrm{m})^{2}$ and $20(\mathrm{mV} / \mathrm{m})^{2}$. The total power of the background hiss for the Cluster LHCs thus seems to be slightly lower than it is for sounding rocket rocket LHC observations.

\section{Observations with four Cluster satellites}

As the Cluster mission consists of four satellites it is possible to compare the data from all of them to see how the occurrence of LHCs in a region is changing with time. In the data from 29 August 2002, the LHCs are so clear in the electric field measurements alone that we can identify a region where they occur not only in the data from spacecraft 1 and 3 , where high resolution spacecraft potential measurements are available, but also in the data from the other two spacecraft where we only have electric field data. While the availability of electric field data alone invalidates our identification criteria for individual LHCs, we can certainly say that they do occur in a region of space when the E-field data from spacecraft 2 and 4 show signatures similar to those clearly correlating with density depletions in the data from spacecraft 1 and 3 (see Sec. 2.2). This means that the region can be identified with all four spacecraft.

The upper panel of Fig. 12 is a timeline of the data for all four spacecraft, where the narrow line means that we have electric field data with high resolution and the thick line means that there are identified LHCs in the region. We see that the first identified LHC in the spacecraft 3 data is more than half an hour later than the last identified LHC for spacecraft 1 . We may now look at the lower panel of Fig. 12, where the orbits of the spacecraft are indicated in terms of invariant latitude and MLT. We clearly see that the identified LHCs in this time interval are confined in a small region between $68^{\circ}$ and $73^{\circ}$ invariant latitude. Note that spacecraft 1 and 2 show the existence of LHCs already at the beginning of the time interval. The conclusion from this example is that the existence of LHCs in a region in space seems to be a stable feature, at least on the time scale of hours. The stability of the individual LHCs cannot be determined from this example, except that they are at least stable on the time it takes for a satellite to pass $(\sim 0.2 \mathrm{~s})$, which is about the time scale of an ion gyro period. In this sense, the existence of LHCs seem to be more a spatial phenomenon than a temporal.

\section{Conclusions}

A major point is that Lower Hybrid Cavities are a common phenomenon also in the inner magnetosphere at altitudes between 10000 and $35000 \mathrm{~km}$. During 87 Cluster (27 Viking) hours of observation, a total of 535 (166) LHCs were found, giving an average of about 6 cavities per hour for all spacecraft. Previous studies have concentrated on altitudes below $2000 \mathrm{~km}$, and a recent study has shown that the properties of the LHCs are very similar at high and low altitudes, even in detail (Tjulin et al., 2003). Other important conclusions from this study are:

- The LHCs we study are found at invariant latitudes from the auroral region, including the dayside cusp, down to the plasmapause. This is consistent with earlier observations at lower altitudes.

- Comparing one Freja orbit at low altitude to our Viking and Cluster data sets, we find that the occurrence frequency of LHCs with a given density depletion does not scale with the flux tube radius. In this sense, LHCs seem somewhat less common at high altitudes.

- It is not clear how this different occurrence frequency should be interpreted in physical terms, as no complete theory is available for LHC formation and dynamics in absolute terms. It may be that the density depletion of the LHCs should scale with altitude in such a way that the identification criteria used in different studies cause 
us to identify a smaller fraction of the LHCs at high altitude than in the ionosphere. However, the difference in occurrence frequency is so big that it is hard to believe this to be a dominating effect. If our result on the occurrence frequency indeed can be interpreted as LHCs being less common at higher altitudes than what is expected from simple scaling with the magnetic field, it indicates that LHCs generally do not extend all the way from the ionosphere up to Cluster altitudes $(35000 \mathrm{~km})$. This is the first observationally derived upper bound on the parallel extension of LHCs.

- The width of the LHCs is a few times the ion gyroradius, consistent with observations at low altitudes.

- The estimated depth of the density depletions vary with altitude, being larger around $1000 \mathrm{~km}$ (sounding rockets, 10-20\%), smaller around 1500-13000 km (Freja and Viking, a few percent) and again larger at altitudes of 20000-35000 km (Cluster, 10-20\%).

- The LHCs in the inner magnetosphere are situated in regions with background electrostatic hiss in the lower hybrid frequency range. The total power of this hiss seems to be slightly smaller at Cluster altitudes (20000$35000 \mathrm{~km}$ ), compared to sounding rocket data (around $1000 \mathrm{~km})$

- Observations by the four Cluster satellites show that the existence of LHCs in a region seems to be stable, at least on a time scale of an hour. Observations of many individual LHCs show that they are stable at least on the time scale of $0.2 \mathrm{~s}$ (about one ion gyro period), in agreement with Lynch et al. (1999) and Knudsen et al. (1999). The LHC phenomenon seems to be more spatial than temporal.

Acknowledgements. This work was partially supported by the European Community's Human Potential Programme under contract HPRN-CT-2001-00314. The background magnetic field data on Cluster were provided by the FGM instrument team (A. Balogh).

Topical Editor T. Pulkkinen thanks two referees for their help in evaluating this paper.

\section{References}

Bahnsen, A., Jespersen, M., Ungstrup, E., Pottelette, R., Malingre, M., Decreau, P. M. E., Hamelin, M., de Feraudy, H., Perraut, S., and Pedersen, B. M.: First Viking results: High frequency waves, Phys. Scripta, 37, 469-474, 1988.

Balogh, A., Dunlop, M. W., Cowley, S. W. H., Southwood, D. J., Thomlinson, J. G., Glassmeier, K. H., Musmann, G., Lühr, H., Buchert, S., Acuña, M. H., Fairfield, D. H., Slavin, J. A., Riedler, W., Schwingenschuh, K., and Kivelson, M. G.: The Cluster Magnetic Field Investigation, Space Sci. Rev., 79, 65-91, 1997.

Cornilleau-Wehrlin, N., Chauveau, P., Louis, S., Meyer, A., Nappa, J. M., Perraut, S., Rezeau, L., Robert, P., Roux, A., de Villedary, C., de Conchy, Y., Friel, L., Harvey, C. C., Hubert, D., Lacombe, C., Manning, R., Wouters, F., Lefeuvre, F., Parrot, M.,
Pinçon, J. L., Poirier, B., Kofman, W., and Louarn, P.: The Cluster Spatio-Temporal Analysis of Field Fluctuations (STAFF) Experiment, Space Sci. Rev., 79, 107-136, 1997.

Dovner, P. O., Eriksson, A. I., Boström, R., Holback, B., Waldemark, J., Eliasson, L., and Boehm, M.: The occurence of lower hybrid cavities in the upper ionosphere, Geophys. Res. Lett., 24, 619-622, 1997.

Eriksson, A. I. and Boström, R.: Wave measurements using electrostatic probes: Accuracy evaluation by means of a multiprobe technique, in: Measurement techniques in space plasmas, edited by Borovsky, J., Pfaff, R. and Young, D., vol. 103 of AGU Geophysical Monograph, AGU, 147-153, 1998.

Eriksson, A. I., Holback, B., Dovner, P. O., Boström, R., Holmgren, G., André, M., Eliasson, L., and Kintner, P. M.: Freja observations of correlated small-scale density depletions and enhanced lower hybrid waves, Geophys. Res. Lett., 21, 1843-1846, 1994.

Eriksson, A. I., Mälkki, A., Dovner, P. O., Boström, R., Holmgren, G., and Holback, B.: A statistical study of auroral solitary waves and weak double layers, 2. Measurement accuracy and ambient plasma density, J. Geophys. Res., 102, 11 385-11 398, 1997.

Escoubet, C. P., Schmidt, R., and Goldstein, M. L.: Cluster - Science and mission overview, Space Sci. Rev., 79, 11-32, 1997.

Escoubet, C. P., Fehringer, M., and Goldstein, M.: The Cluster mission, Ann. Geophys., 19, 1197-1200, 2001.

Gustafsson, G., Boström, R., Holback, B., Holmgren, G., Lundgren, A., Stasiewicz, K., Åhlén, L., Mozer, F. S., Pankow, D., Harvey, P., Berg, P., Ulrich, R., Pedersen, A., Schmidt, R., Butler, A., Fransen, A. W. C., Klinge, D., Thomsen, M., Fälthammar, C.G., Lindqvist, P.-A., Christenson, S., Holtet, J., Lybekk, B., Sten, T. A., Tanskanen, P., Lappalainen, K., and Wygant, J.: The electric field and wave experiment for the Cluster mission, Space Sci. Rev., 79, 137-156, 1997.

Høymork, S. H., Pécseli, H. L., Lybekk, B., Trulsen, J., and Eriksson, A. I.: Cavitation of lower hybrid waves in the Earth's ionosphere: A model analysis, J. Geophys. Res., 105, 18 519-18 535, 2000 .

Hultqvist, B.: The Swedish satellite project Viking, J. Geophys. Res., 95, 5749-5752, 1990.

Kintner, P. M., Vago, J., Chesney, S., Arnoldy, R. L., Lynch, K. A., Pollock, C. J., and Moore, T. E.: Localized lower hybrid acceleration of ionospheric plasma, Phys. Rev. Lett., 68, 2448-2451, 1992.

Kjus, S. H., Pécseli, H. L., Lybekk, B., Holtet, J., Trulsen, J., Lühr, H., and Eriksson, A.: Statistics of the lower hybrid wave cavities detected by the Freja satellite, J. Geophys. Res., 103, 26633 26647, 1998.

Knudsen, D. J., Wallis, D. D., and James, H. G.: Tethered two-point measurements of solitary auroral density cavities, Geophys. Res. Lett., 26, 2933-2936, 1999.

Knudsen, D. J., Bock, B. J. J., Bounds, S. R., Burchill, J. K., Clemmons, J. H., Curtis, J. D., Eriksson, A. I., Koepke, M. E., Pfaff, R. F., Wallis, D. D., and Whaley, N.: Lower-hybrid cavity density depletions as a result of transverse ion acceleration localized on the gyroradius scale, J. Geophys. Res., 109, A04 212, doi:10.1029/2003JA010089, 2003.

LaBelle, J., Kintner, P. M., Yau, A. W., and Wahlén, B. A.: Large amplitude wave packets observed in the ionosphere in association with transverse ion acceleration, J. Geophys. Res., 91, 71137118, 1986.

Lynch, K. A., Arnoldy, R. L., Kintner, P. M., Schuck, P., Bonnell, J. W., and Coffey, V.: Auroral acceleration from lower hybrid solitary structures: A summary of sounding rocket observations, 
J. Geophys. Res., 104, 28 515-28 534, 1999.

Pécseli, H. L., Iranpour, K., Holter, Ø., Lybekk, B., Holtet, J., Trulsen, J., Eriksson, A. I., and Holback, B.: Lower-hybrid cavities detected by the Freja satellite, J. Geophys. Res., 101, 52995316, 1996.

Pedersen, A.: Solar wind and magnetosphere plasma diagnostics by spacecraft electrostatic potential measurements, Ann. Geophys., 13, 118-129, 1995.

Pedersen, A., Décréau, P., Escoubet, C.-P., Gustafsson, G., Laakso, H., Lindqvist, P.-A., Lybekk, B., Masson, A., Mozer, F., and Vaivads, A.: Four-point high time resolution information on electron densities by the electric field experiments (EFW) on Cluster, Ann. Geophys., 19, 1483-1489, 2001.

Pinçon, J.-L., Kintner, P. M., Schuck, P., and Seyler, C. E.: Observation and analysis of lower hybrid solitary structures as rotating eigenmodes, J. Geophys. Res., 102, 17 283-17 296, 1997.
Pottelette, R., Treumann, R. A., and Dubouloz, N.: Generation of auroral kilometric radiation in upper hybrid wave - lower hybrid soliton interaction, J. Geophys. Res., 97, 12 029-12 044, 1992.

Schuck, P. W., Bonnell, J. W., and Kintner Jr., P. M.: A review of lower hybrid solitary structures, IEEE Trans. Plasma Sci., 31, 1125-1177, 2003.

Shapiro, V. D.: Modulational interaction of the Lower-hybrid waves with a kinetic-Alfvén mode, Phys. Rev. Lett., 81, 3415-3418, 1998.

Tjulin, A., Eriksson, A. I., and André, M.: Lower hybrid cavities in the inner magnetosphere, Geophys. Res. Lett., 30, 1364, doi:10.1029/2003GL016915, 2003.

Tjulin, A., Eriksson, A. I., and André, M.: Localization of wave fields in lower hybrid cavities, Ann. Geophys., 22, 2951-2959, 2004.

Vago, J. L., Kintner, P. M., Chesney, S. W., Arnoly, R. L., Lynch, K. A., Moore, T. E., and Pollock, C. J.: Transverse ion acceleration by localized lower hybrid waves in the topside auroral ionosphere, J. Geophys. Res., 97, 16 935-16957, 1992. 